Themenheft Nr. 45:

Pädagogisches Wissen im Lichte digitaler und datengestützter Selbstoptimierung Herausgegeben von Estella Ferraro, Sabrina Schröder und Christiane Thompson

\title{
Editorial: Pädagogisches Wissen im Lichte digitaler und datengestützter Selbstoptimierung
}

\author{
Eine Einleitung \\ Sabrina Schröder ${ }^{1}$ (D), Christiane Thompson ${ }^{2}$ (D) und Estella Ferraro² (D) \\ ${ }^{1}$ Universität Kassel \\ 2 Johann Wolfgang Goethe-Universität Frankfurt
}

\section{Digitalisierung als «Strategie»}

In ihrem Strategiepapier «Bildung in der digitalen Welt» tritt die Kultusministerkonferenz (KMK) dafür ein, das Bildungssystem in Deutschland mit «Hochdruck und nachhaltig» (KMK 2016, 8) als Teil der «digitale[n] Revolution» (ebd.) zu verstehen und über Empfehlungen und Anforderungen die Akteurinnen und Akteure des Bildungswesens in digitale Entwicklungsmöglichkeiten und -notwendigkeiten zu involvieren. Digitalisierung wird dabei einerseits als «dynamischer Prozess» (ebd., 61) dargestellt, der durch permanente Weiterentwicklungen und «kurze Innovationszyklen» (ebd., 21) Mobilität und Flexibilisierung, Wandel und stetes Lernen gesellschafts- und bildungspolitisch erfordert und herausfordert. Andererseits wird Digitalisierung in den Kontext eines Kompetenzerwerbs gestellt, über den allererst möglich werde, auf die sich stetig verändernden Bedingungen von flexiblen Lern- und Arbeitswelten angemessen zu reagieren. Auf den digitalen Innovationsdruck, der sich auf alle Lebensbereiche auswirke, müsse vor allem im Bildungssystem «schneller reagiert werden, so im analogen Papier des Bundesministeriums für Bildung und Forschung (BMBF) mit dem Titel «Bildungsoffensive für die digitale Wissensgesellschaft» (BMBF 2016, 2).

Begriffe wie «digitale Revolution` machen die Grundsätzlichkeit deutlich, mit der in den Strategiepapieren der KMK und des BMBF die Veränderungsnotwendigkeiten und -potenziale im Lichte von Digitalisierung behauptet, gefordert und beschworen werden. Aus aktuellen Debatten zum Bildungssystem liessen sich zahlreiche Papiere anführen, die - auch unter den Eindrücken der Corona-Pandemie - in ähnlicher Weise Innovationen von Bildung im Horizont von Digitalisierung als zentrale Maxime der Weiterentwicklung des Bildungssystems verstehen (vgl. z. B. das Hagener Manifest (HM 2020) zu «New Learning»).

Die oben bereits angesprochene Mehrstelligkeit einer Innovation von Bildung durch Digitalisierung, angesichts von Digitalisierung sowie im Medium von Digitalisierung als Prozesslogik ist ein wesentlicher Bestandteil der Rhetorik dieser 
Strategiepapiere. Sie dient uns im Folgenden als Hinführung zu einer der Leitthesen dieses Themenheftes: Entwicklungen der Digitalisierung und Datafizierung von Bildung bzw. Bildungsinstitutionen vollziehen sich sehr stark über ein Dispositiv der Selbstoptimierung, das sich durch ein komplexes Bündel von digitalen Technologien

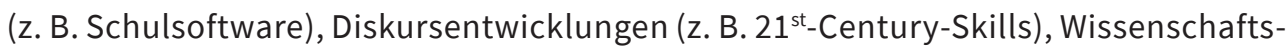
feldern (z. B. Learning Analytics), Menschenbildern (z. B. Transhumanismus) sowie Selbstpraktiken (z. B. Selftracking) konstituiert. Die Wirksamkeit dieses Dispositivs entfaltet sich dadurch, dass alle Akteurinnen und Akteure, Prozesse und auch die pädagogischen Zielbestimmungen unter die Forderung der Ausschöpfung von Qualität und Einsatz gestellt werden. Die qualitätssteigernden Veränderungsanforderungen der Digitalisierungsoffensive haben einen systemischen Charakter.

Erstens: Die Optimierung der Lernenden wird dort greifbar, wo deren «Verantwortung» für die eigenen Lernprozesse eingefordert wird (KMK 2016, 12ff.). Die Verantwortungsübernahme wird mit verschiedenen Motiven der Selbstoptimierung verschränkt, z. B. mit dem Fokus auf ein «kreative[s] und kritische[s]» Lernen (ebd., 13), ein Lernen also, das über den Status quo von Wissen hinausgeht. Ihr Lernen optimieren die Lernenden ausserdem durch Prozessbewusstsein, Organisation und Commitment. Unterstützt werden sie dabei durch die institutionelle Verankerung neuer Möglichkeiten zur Individualisierung des Lernens (vgl. ebd.), was auf eine Neuausrichtung des Bildungsauftrags verweist.

Die Optimierung richtet sich demnach - zweitens - auf die Institution. Schulen, Hochschulen, Kindertagesstätten etc. - alle Bildungseinrichtungen stehen unter der Forderung, ein erfolgreiches Lernen für alle zu ermöglichen. Letzteres soll über differenzierte bzw. heterogenitätssensible Lernräume möglich werden, d. h. über Lernumgebungen, die genau auf die Adressatinnen und Adressaten im Einzelnen ausgerichtet sind. Sie bilden sozusagen das Komplement des optimierten Lernens. Bezogen auf die Optimierung der Institution gibt es allerdings noch einen weiteren wichtigen Grundzug: Bildungsinstitutionen werden als Optimierungsmotoren inszeniert. Ihnen kommt eine Innovationskraft dadurch zu, dass sie auf die «digitale Revolution > des Lernens nicht nur reagieren, sondern gewissermassen prospektiv und selbstgesteuert Eigeninitiative ergreifen. So werden im KMK-Papier bestimmte Schulen zu strategischen Akteurinnen der Digitalisierung: «lm Rahmen ihrer schulischen und pädagogischen Gestaltungsmöglichkeiten greifen innovativ arbeitende Schulen solche Veränderungen von sich aus auf und werden somit zum Wegbereiter für andere» (KMK 2016, 11). Für die Hochschulen liessen sich zahlreiche analoge Formulierungen angeben.

An dieser Stelle dürfen drittens die Lehrenden nicht ausgelassen werden, die in den Strategiepapieren ebenfalls zu Akteurinnen und Akteuren der Optimierung werden: Sie sollen durch kontinuierliche Weiterbildungsmassnahmen «Selbstverantwortung für den eigenen Kompetenzzuwachs» (ebd., 27) übernehmen - einerseits im 
Sinne einer Ausbildung allgemeiner Medienkompetenz; andererseits sollen sie bezogen auf ihre «fachlichen Zuständigkeiten zugleich «Medienexperten` werden» (ebd., 24f.). Lehrende bleiben also nicht nur bezüglich ihres Wissens in optimaler Weise up to date; als Expertinnen und Experten für Medien und deren Gebrauch werden sie zu Botschafterinnen und Botschaftern eines innovativen Umgangs mit Wissen. Sie erweitern symbolisch und praktisch die Reichweite digitalisierter bzw. medial gestützter Wissensvermittlung und eröffnen damit Spielräume der Teilhabe für die Lernenden und die Gestaltung des Lernens.

Was unter Bezugnahme auf das Strategiepapier der KMK einführend deutlich wird, ist, wie Digitalisierung für die Weiterentwicklung des Bildungssystems symbolisch verbindlich gemacht wird und wie dies als mehrstelliges Projekt der Optimierung aufgesetzt wird. Die Lernenden, das Lernen, die Lehrenden, aber auch die Vorstellungen vom Lernen, die institutionellen Routinen, die medialen Architekturen und Lernräume werden unter die Anforderung der Veränderung und Qualitätssteigerung gestellt, wobei Digitalität gleichermassen Erfordernis, Instrument und Ziel des Ganzen ist.

In welcher Weise sich damit die Formen und Konstitutionsbedingungen pädagogischen Wissens verändern und welche Konsequenzen dies für das pädagogische Selbstverständnis in sowohl institutionell verankerten als auch ausserinstitutionellen pädagogischen Kontexten und Handlungsfeldern haben kann, sind zentrale Fragen des vorliegenden Publikationsprojektes. Leitendes Erkenntnisinteresse dieses Heftes ist die Analyse von Formen und Praxen pädagogischer Optimierung im Lichte von Digitalisierung und Datafizierung, etwa als individualisierter Selbstbezug über Selftracking-Praktiken, über die Aufforderung zur Optimierung in der Prüfung oder über eine potenzielle Optimierbarkeit professionellen pädagogischen Handelns durch datengestützte Tools. Im Folgenden gehen wir kurz auf die Ausgangsidee und Entwicklung des Heftes ein.

\section{Zu diesem Themenheft}

Dieses Themenheft entstand aus der gemeinsamen Arbeit an einem Symposium, das ursprünglich auf dem Kongress der Deutschen Gesellschaft für Erziehungswissenschaft (DGfE) im März 2020 stattfinden sollte. Der Kongress, der kurzfristig pandemiebedingt abgesagt werden musste, stand unter der Kategorie der «Optimierung» und sollte unter anderem thematisieren, wie Optimierungsdispositive in die Pädagogik bzw. Erziehungswissenschaft einsickern und dadurch Transformationen des Pädagogischen in Gang setzen (vgl. DGfE 2020). Über die Zusammenarbeit des geplanten Symposiums hinaus erweiterte sich der Kreis der Kolleginnen und Kollegen, um verschiedene Ebenen und Bezüge des Dispositivs einer digitalen bzw. datenbezogenen Selbstoptimierung einzuholen und für das Themenheft fruchtbar zu machen. 
Im Herbst 2020 fand ein gemeinsamer Autorinnen- und Autorenworkshop statt, auf dem die beteiligten Kolleginnen und Kollegen ihre Perspektive auf eine datengestützte Selbstoptimierung vorstellten. Gemeinsam wurden unterschiedliche Nuancen von ‘Optimierung) und deren Implikation für die Vorstellung von pädagogischem Wissen diskutiert. Im Rahmen des Workshops wurde zudem eine erste Qualitätsprüfung vorgenommen, auch im Hinblick auf die wechselseitigen Bezüge der Beiträge untereinander. Über ein Review durch die Herausgeberinnen erfolgte eine weitere Feedbackrunde nach Einreichung der Beiträge. Auf dieser Grundlage wurde der einleitende Beitrag erstellt, dem die Aufgabe zukommt, den Rahmen abzustecken, unter dem die Beiträge ihren Blick auf die Veränderung pädagogischen Wissens richten.

Dazu konturieren wir zunächst den Begriff der Optimierung, welcher seit gut 15 Jahren in den Sozial- und Kulturwissenschaften als analytisches Konzept in Verwendung ist. Ohne hier eine erschöpfende Bestandsaufnahme geben zu können, wollen wir die erkenntnistheoretische und erkenntnispolitische Valenz dieses Konzepts herausarbeiten und im Verhältnis dazu die Rezeption bzw. Verwendung in der Erziehungswissenschaft beleuchten. Wir verbinden diese Bestandsaufnahme mit Erkenntnissen aus den Critical Data Studies und jüngsten Ergebnissen der Policy Studies zu den Transformationen von Bildung im Lichte eines knowledge-intensive capitalism. Am Ende dieser thematischen Hinführung geben wir einen Vorblick auf die Beiträge des Themenheftes.

\section{Zum Konzept der Optimierung}

In der Alltagssprache dominiert ein operativer Begriff der Optimierung. Bestehende Verfahren, Prozesse und Routinen werden verändert, um ihren Vollzug, ihre Wirkung und Ergebnisse zu verbessern oder zu steigern. Der Begriff fokussiert auf das bessere Zusammenspiel von Elementen in (ökonomischen) Prozessen; Optimierung wird durchaus als dynamisch aufgefasst, weil sie unbekannten Parametern bzw. Bedingungen unterliegt. Betriebsabläufe werden ebenso optimiert wie Produktionswege oder die Rekrutierung von Mitarbeiterinnen und Mitarbeitern. Diese «Optimierung» lässt sich mathematisch formalisieren, insofern als zu einer Funktion bzw. einer Problemstellung ein Maximalwert (oder auch ein Minimum) zu bestimmen ist. Es geht dann z. B. darum, die Funktionsfähigkeit einer Software in einem Betrieb auszuweiten, unter Berücksichtigung der Grenzen ihrer Kompatibilität oder auch der Fähigkeiten der Mitarbeitenden, diese zu bedienen. Optimierungsaufgaben sind konkret und systemisch, indem sie auf die Operativität des Ganzen gerichtet sind.

Der Begriff der Optimierung, wie er seit 15-20 Jahren in den Sozial- und Kulturwissenschaften verwendet wird, greift durchaus das Systemische und Operative auf. Die Pointe des Konzepts liegt allerdings in einem anderen Aspekt, nämlich in seiner Bedeutung als 〈Subjektivierungsform〉. Das Verdienst, 〈Optimierung〉 als ein 
dominantes Konzept gegenwärtiger Selbstbeschreibungen oder Selbstverhältnisse herausgestellt zu haben, ist wesentlich Ulrich Bröckling zuzuschreiben. In seiner Studie «Das unternehmerische Selbst» hat Bröckling im Jahr 2007 aus einer Analyse gegenwärtiger Ratgeber der Management-Literatur die Figur des «unternehmerischen Selbst» verdichtet, das dazu angehalten ist, sich als Projekt zu denken und das eigene Wirken einer permanenten Veränderung zu unterstellen. Entscheidend sind dabei Selbstverhältnissetzungen, die unabschliessbar sind und die dem Anspruch der permanenten Neukreation unterliegen (vgl. Bröckling 2007, 35). Bröckling differenziert zwischen drei Varianten der Optimierung, die sich im Hinblick auf ihre Prozessualität und (Un-)Abschliessbarkeit unterscheiden lassen (vgl. Bröckling 2013, 4).

Erstens lässt sich Optimierung verstehen als ein Streben nach Vervollkommnung im Hinblick auf ein Ziel, das sich zwar nie vollständig erreichen lässt, von dem das Subjekt jedoch mehr oder weniger weit entfernt sein kann, sodass das Ziel selbst zum Motor der Anstrengung und der Arbeit an sich selbst wird. Optimierung wird hierbei als «Perfektionierung» gefasst (ebd.). Davon unterscheidet Bröckling zweitens die Optimierung als eine Ausrichtung auf den Weg (statt auf das Ziel) und auf die Quantifizierungsmöglichkeit der Anstrengung. Optimierung wirkt hier wie eine 〈Richtungsangabe - als eine Fort- und Weiterentwicklung, die kein Ende kennt. In dieser Form der Optimierung geht es um eine kontinuierliche «Steigerung» (ebd.), der Fort- und Weiterbildung, des lebenslangen Lernens etc. Die dritte Variante stellt noch stärker den relationalen Charakter von Optimierung in den Mittelpunkt - ihr Modus ist der «Wettbewerb» (ebd.), ihr Massstab ist der Vergleich und die Konkurrenz. Optimierung versteht sich in dieser Variante «vom anderen her), als etwas, wovon es sich abzugrenzen gilt. Bröckling spricht an anderer Stelle von einem «kreative[n] Imperativ», der «zur permanenten Abweichung» nötigt (Bröckling 2007, 170). Wenn hier das Ziel darin besteht, in einer Welt der Wahlen gewählt zu werden (vgl. Reckwitz 2006, 451), ist die kontinuierliche und damit selbstverändernde Arbeit an sich eine Notwendigkeit zur Erreichung dieses Ziels. Das Subjekt muss sich permanent neu erfinden, weil - im Horizont eines Marktes von Möglichkeiten und Gelegenheiten - von überall her Konkurrenz droht. ${ }^{1}$

Es wäre jedoch verfehlt oder voreilig, dieses Unternehmertum als Zwangsverhältnis zu bestimmen, denn das unternehmerische Selbst operiert unter dem Anspruch von Selbststeuerung, Autonomie und Selbstverwirklichung (Mayer und Thompson 2013). Wie der Selbstbezug bzw. dieses Selbstverhältnis zu denken ist, erschliesst sich an der spezifischen Logik des Optimierungsbegriffs, in dem ein doppelter Bezug von Unterworfenheit und Freiheit in einen paradoxen Subjektivierungsprozess einmündet: «Ein Subjekt zu werden ist etwas, dem niemand entgeht und das zugleich

1 Kreativität erscheint in dieser Perspektive als eine Form des Kontingenzmanagements. Abgeblendet werden muss dabei, dass sich kreative Akte nicht intentional erzeugen lassen (vgl. Bröckling 2007, 153) und dass ein potenzielles Scheitern als Schatten über all den Bemühungen liegt, weil der Erfolg der jeweiligen 〈Abweichung〉 (d. h. ihr Marktpotenzial) nicht kalkulierbar ist. 
niemandem gelingt» (Bröckling 2007, 30). Die Paradoxalität wird in der Unbestimmtheit des Ziels (das Optimum) offenbar, aber auch dadurch, dass das Subjekt im Horizont dieser zu bestimmenden Zielsetzung auf sich selbst zurückgeworfen wird. Die Aufforderung «Werde du selbst» (ebd., 42) spannt die Selbstverbesserung, -verantwortung, -innovation, -steigerung und -kreativität zwischen einem Ist- und Soll-Zustand auf, wobei beide Zustände zunehmend ihre Kontur verlieren.

Bei aller Getriebenheit und Unbestimmbarkeit steht Optimierung für Freiheit, Autonomie und Wahl, weil sich das Subjekt über die Aufforderung zur Optimierung als gestaltbar erfahren kann und diese Gestaltbarkeit als einen Handlungsspielraum oder eine Möglichkeit für sich selbst ausmachen kann. Mit verschiedenen optimierenden Technologien kann das Selbst, anders gesagt, seinen Wünschen, Bedürfnissen, Zielen nachgehen, um auf diese Weise «einen gewissen Zustand des Glücks» (Foucault 1993, 26) zu erlangen. Als Beispiel hierfür könnte die Gesundheitsvorsorge angeführt werden: Sich heutzutage als «gesund» zu verstehen, ist kaum mehr über die Abwesenheit von Krankheit zu fassen. Demgegenüber gilt es, individuell den Gestaltungsspielraum des eigenen Wohlbefindens zu erschliessen. Wearables wie die Smartwatch erlauben völlig neue Einblicke in den eigenen Körper: Sie behalten die Sauerstoffsättigung des Blutes im Blick, überwachen und analysieren den Schlaf oder optimieren über Zyklusmonitoring und Temperaturmessungen die Chancen zur Verwirklichung eines Kinderwunsches. Mit Blick auf das Dispositiv der Selbstoptimierung liesse sich davon sprechen, dass Prävention an die Technologien des Selbst gekoppelt wird, d. h. Prävention ist keineswegs «nur als Vermeidung zu verstehen; vielmehr wird sie in den Horizont der Verbesserung des Selbst gestellt.

Bei der Selbstoptimierung geht es also immer auch um das Entdecken neuer Gelegenheiten und Möglichkeiten, sich zu entwickeln. Dem entspricht, so Bröckling unter Bezugnahme auf Boltanski und Chiapello (2003), die Aufwertung des Projektbegriffs, der die Tätigkeits- und Wirkungsprofile von Aktivitäten verunklart und der die Grenzen von Arbeit und Nicht-Arbeit oder Produktion und Konsumption verwischt (Bröckling 2007, 260ff.). Die projektförmige Arbeit gilt als «Königsweg zu mehr Innovationsfähigkeit und Flexibilität» (Bröckling 2005, 376) und auch zu mehr «Selbstverantwortung» (ebd., 381). Selbstverantwortlich soll das Subjekt auf die sich ständig ändernden Lebens-, Lern- oder Arbeitsbedingungen reagieren, indem es sich flexibel neu organisiert. Es soll sich selbst als (Projekt) begreifen, das es zu optimieren gilt.

In ihrer kürzlich veröffentlichten Studie hat Röcke (2021) herausgearbeitet, wie weitgehend die Projekte und Prozesse der Selbstoptimierung zum «inhärente[n] Bestandteil eines auf Leistung, Erfolg und Selbstverwirklichung ausgerichteten Wertekanons gegenwärtiger westlicher Gesellschaften» geworden sind (ebd., 14). Die Arbeit an sich selbst, die Strategien und Techniken, die dazu eingesetzt werden, sich selbst ‘zu erkennen`, werden immer mehr verfeinert, ebenso wie die Sphären dessen sich ausweiten, was einer Verbesserung unterzogen werden kann (vgl. Mayer und Thompson 2013). 
Die soziologischen Studien zum unternehmerischen Selbst wie überhaupt das interdisziplinäre Programm einer Subjektivierungsforschung (Ricken, Casale, und Thompson 2019) sind im engen Rückbezug auf Foucaults Problemstellung der Regierung bzw. Menschenführung zu denken (Foucault 2004). Gegen die Vorstellung einer monolithischen Staatsmacht setzte Foucault die Analyse von vielfältigen Macht-Wissens-Beziehungen, die mit der Neuzeit zur Führung und Selbstführung der Einzelnen wirksam werden. Foucaults Analytik diskursiver Praktiken und seine genealogischen Studien der Macht ermöglichten zum einen, die Entwicklung der Humanwissenschaften und damit auch der Pädagogik unter dieser Perspektive zu diskutieren und aufzunehmen. Die Gestaltung pädagogischer Verhältnisse und Prozesse in Theorie und Praxis wurde damit als Antwort auf die Frage der Führung der Selbstführungen lesbar gemacht. ${ }^{2}$ Das Foucault'sche Instrumentarium konnte zum anderen für eine «kritische Ontologie der Gegenwart> (vgl. auch Thompson 2004) zum Einsatz gebracht werden - gerade auch im Hinblick auf den aktuellen Umbau des Bildungssystems. Bevor wir diesen analytischen Einsatzpunkt im Folgenden aufgreifen, wollen wir zunächst auf die Mehrdeutigkeit des Optimierungskonzepts in der Erziehungswissenschaft eingehen.

\section{Ambivalenzen von «Optimierung» im erziehungswissenschaftlichen Terrain}

Dass die Verwendung des Optimierungsbegriffs in der Erziehungswissenschaft von Ambivalenzen geprägt ist, kann anhand der Themenbeschreibung des DGfE-Kongresses erläutert werden, auf dem wir unsere Beiträge ursprünglich vorstellen wollten. In der Beschreibung des Tagungsthemas ist von Optimierung als «leitende[r] Strategie» und «elementare[m] Topos» der Erziehungswissenschaften die Rede (DGfE 2020). Darüber hinaus wird Optimierung als eine der «zentralen Signaturen der Gegenwart» (ebd.) und damit als gesellschaftlich relevant bestimmt. So hoch die wissenschaftliche und gesellschaftliche Relevanz eingeschätzt ist, so unterbestimmt bleibt doch «Optimierung» - vor allem in ihrer kategorialen Bedeutung für die Erziehungswissenschaft. Mit anderen Worten: Wie sich Erziehung, Bildung und Optimierung zueinander verhalten und welche Unterscheidungen, Paradigmen oder (wissenschaftlichen) Felder heranzuziehen sind, um dieses Verhältnis zu bestimmen, bleibt in der Ankündigung unklar.

Diese Unbestimmtheit lesen wir als eine Konsequenz der disziplinären und disziplinpolitischen Entwicklung der Erziehungswissenschaft, die in einem engen Zusammenhang einer Bildungsexpansion zu sehen ist, die gleichermassen von bildungsökonomischen und gesellschaftspolitischen Motiven getragen war. Wenn in disziplingeschichtlichen Bestandsaufnahmen von einer Versozialwissenschaftlichung

2 Darauf werden wir zu sprechen kommen. Eine wichtige Studie, welche die Bedeutung von Bildung in diesem Horizont aufgreift, ist Norbert Rickens Monografie «Die Ordnung der Bildung» (Ricken 2006). 
der Disziplin gesprochen wird, geraten jene Bezüge aus Bildungspolitik und Praxis aus dem Blick, welche die Konstitution des erziehungswissenschaftlichen Feldes mitbestimmen (vgl. Thompson 2021). Letzteres lässt sich nicht verstehen, ohne den Aufstieg jener transnationalen Akteurinnen und Akteure mitzudenken, die Aspekte von Bildung und Bildungsentwicklung in den letzten Dekaden zu einer globalen Angelegenheit des Wettbewerbs und der Anhäufung von Humankapital gemacht haben: die OECD, die Weltbank, aber auch die EU, Stiftungen, z. B. Bertelsmann, oder international aufgestellte Firmen, wie Pearson.

Diese Akteurinnen und Akteure bespielen eine bildungspolitische Bühne dadurch, dass sie die Erforschung von Bildungs- und Lernprozessen vollkommen neu ausrichten. Ein sprechendes Beispiel hierfür ist der OECD-Bericht «Understanding the Brain: The Birth of a Learning Science» (OECD 2002), in dem das Gehirn zum Subjekt des Lernens gemacht wird. Durch diese epistemologische Verschiebung wird die Erziehungswissenschaft auf die Ermöglichung eines hirngerechten Lernens im Zeichen von Evidenz und Effizienz verpflichtet. ${ }^{3}$ Was soll damit anderes gemeint sein als das Versprechen eines optimalen Lernens, das die ‘Reibungsverluste` durch traditionelle Lehrformate, Lehrende oder Theorien verhindert? Die Veränderungen, die sich aktuell vollziehen, betreffen nicht nur den Umbau von Bildungsinstitutionen. Sie betreffen gerade auch die Erziehungswissenschaft im Hinblick auf ihre Erkenntnisinteressen, ihre leitenden Begriffe und Theorien wie auch ihre Methoden zur Erforschung von Lern- und Bildungsprozessen.

Bellmann hatte die Veränderungen der Erziehungswissenschaften unter der Chiffre einer Entwicklung hin zur Bildungsforschung beschrieben, die in ein komplementäres Verhältnis zur Bildungspolitik trete. Nach Bellmann ist die Bildungsforschung durch ihre Ausrichtung auf Outputsteuerung zu einer Verbündeten der Bildungspolitik geworden (Bellmann 2006; vgl. auch Bellmann in Bellmann et al. 2020). Sicherlich ist diese Beschreibung zu allgemein und es ist in jedem Fall wichtig, diese Charakterisierung nicht als Generalkritik an die Adresse der empirischen Bildungsforschung zu begreifen. ${ }^{4}$ Eine von Bellmann aufgegriffene Figur erscheint uns indes systematisch bedeutsam. Es handelt sich um das kybernetische Modell, das der Weiterentwicklung des Bildungssystems unterlegt wird: Die Bildungspolitik gibt Zielstellungen vor, die nach Anpassungsleistungen im System von der Bildungsforschung überprüft und moderiert werden (Bellmann 2016). Die Erziehungswissenschaft wird an dieser Stelle zum Optimierungsinstrument des bildungspolitischen Handelns.

Ein im Jahr 2020 geplanter erziehungswissenschaftlicher Kongress zu «Optimierung» fasst in sich also eine schon lang andauernde Entwicklung, welche eine Neuformierung und Durchdringung der Erziehungswissenschaft im Kontext von Neuer

3 Casale et al. (2010) haben von einer «Geburt der Lernwissenschaft» und einem «Untergang der Erziehungswissenschaft» gesprochen (Casale et al. 2010).

4 Für eine wichtige innerdisziplinäre Debatte vgl. das Sonderheft der ZfE: «Empirische Bildungsforschung. Der kritische Blick und die Antwort auf die Kritiker» (Baumert und Tillmann 2016). 
Steuerung, Bildungspolitik und Bildungsökonomie einschliesst. Dies - wie auch die Abgrenzung zu einer Erziehungswissenschaft, die nicht mehr auf der Höhe der Zeit gesehen wird - entbirgt auf einen Schlag die Ambivalenz im Programm des Kongresses. Wir haben an dieser Stelle nicht den Raum, die Veränderungen des wissenschaftlichen Feldes der Erziehungswissenschaft eingehender zu beschreiben. Worauf wir aber hinweisen wollen, ist, dass die hier angedeuteten Entwicklungen nicht denkbar wären - ohne Daten. Entscheidend ist u. E., die Umgestaltung von Bildungssystem und Erziehungswissenschaft in einer engen Verbindung mit der Erhebung, Bereitstellung und Verarbeitung von Daten zu begreifen. Hier liegt ein gegenüber der von Bröckling betriebenen Erforschung von Optimierungsprozessen verschobener Einsatzpunkt dieses Themenheftes: Zwar ist und bleibt die Frage zentral, wie Anrufungen der Optimierung adressiert werden; um den Umbau von Bildungsinstitutionen und die dabei eingesetzten Optimierungsprozeduren aufzuschliessen, dürfen indes die eingesetzten Technologien, diskursiven Strategien und ihre Vernetzung im Rahmen eines digitalen Kapitalismus nicht übersehen werden.

\section{Pädagogisches Wissen im Horizont datengetriebener Optimierung}

Wir haben eingangs die Mehrstelligkeit hervorgehoben, mit der Digitalisierung als Strategie der Verbesserung und Optimierung des Bildungswesens verfolgt wird. Im vorangehenden Abschnitt haben wir deutlich gemacht, dass die Erziehungswissenschaft von dieser strategischen Dimensionierung selbst betroffen ist: Sie unterliegt dieser Strategie ebenso wie sie selbst Strategieträgerin von Digitalisierung wird. Von Letzterem zeugt ein wachsendes Feld der Learning Analytics und des Educational Data Mining (Romero et al. 2010). Während wir diese Zeilen verfassen, werden beim BMBF Anträge in einer Förderlinie zur Künstlichen Intelligenz in der Hochschulbildung begutachtet (BMBF 2021). Diese Forschungsförderung dient unmittelbar der Entwicklung neuartiger Instrumente in Hochschulen, um Bildungs- und Lernprozesse durch datengetriebene Prozesse zu optimieren. Dabei erfahren diese Instrumente zugleich eine Implementierung, in deren Folge Bildungsprozesse in eine verbindliche Operationalisierung überführt werden.

Williamson (2017), der die Transformationen des Bildungssystems unter den Bedingungen von Big Data analysiert, zeigt eindrücklich, wie die Produzentinnen und Produzenten von lernbezogenen Dateninfrastrukturen massiv auf das pädagogische Feld Einfluss nehmen, indem sie über die Ausgestaltung des Lernens und die Wissensproduktion bestimmen: «Owning the databases, in other words, is a prerequisite for knowledge production and theory generation, and puts powerful education data science organizations at a competitive advantage over other sites of educational research» (ebd., 117). Mit dem 〈Besitz〉 von Instrumenten und Daten vollzieht sich 
eine Aneignung und Verantwortungsverschiebung der erziehungswissenschaftlichen Wissensproduktion, was die Frage nach den legitimen «Zuständigkeiten` oder Autorisierungsmechanismen in der Generierung pädagogischen Wissens aufwirft.

Es ist danach zu fragen, ob sich auf diese Weise reflexive erziehungswissenschaftliche Auseinandersetzungen um erkenntnispolitische Deutungshoheiten transformieren - bspw. indem Wissen zu einer Angelegenheit des Umgangs mit Daten erklärt wird. Anders gesagt: Wandeln sich erkenntnistheoretische und -politische Auseinandersetzungen im erziehungswissenschaftlichen Terrain zunehmend zu einem organisations- oder akteursbezogenen Wettbewerb um lern- und bildungsbezogenes ¿Datenkapital`? Deutlich wird jedenfalls, dass Daten in steigendem Masse zum elementaren Bestandteil eines (pädagogischen) Macht-Wissens-Komplexes ${ }^{5}$ werden; umgekehrt vollzieht sich die Produktion von pädagogischem Macht-Wissen kaum mehr ausserhalb der «data assemblage» bzw. des Datendispositivs (vgl. Kitchin und Lauriault 2014, 7).

Wir sehen zwischen dem in den Critical Data Studies ausformulierten Datendispositiv und dem Dispositiv der (Selbst-)Optimierung einen engen Zusammenhang: Daten werden erzeugt, weiterverarbeitet und mit Relevanzsetzungen versehen, um Lehrende und Lernende, Bildungsinstitutionen und Lernumgebungen mit einer Wahrheit über sich auszustatten und Verbesserungsmöglichkeiten in Aussicht zu stellen. Durch die auratische Evidenz der Daten lassen sich Unsicherheiten und Risiken managen bzw. beseitigen, Produktivitätssteigerungen erzielen, neue Marktsegmente eröffnen, Wettbewerbe und Wettbewerbsvorteile erzeugen (vgl. ebd.).

Die Daten bilden, bezogen auf Lernen und Bildung, eine Scharnierstelle, weil sie gleichermassen den Output eines Lernereignisses wie auch das Potenzial der Ermittlung zukünftig erfolgreicher Lernwege enthalten. Daten bilden das Substrat der digitalen Optimierung, welches auf der Vermessung und Quantifizierung sowie auf psychometrischen Formen der Expertisierung beruht (vgl. auch Thompson 2019), die unmittelbar und direktiv in die Lernwirklichkeit der Individuen und der Institution übersetzt wird. In diesem Zusammenhang wird von einer «evolution of psychometric forms of expertise and disciplinary practice» gesprochen (Williamson 2017, 106).

Über die datengetriebene Optimierung vollzieht sich nicht nur eine Quantifizierung von Bildung und Lernen, die das Pendant zum Diskurs einer evidenzbasierten Bildungsforschung bildet (Bellmann und Müller 2011). Im engen Zusammenhang damit steht die veränderte Vorstellung eines fluiden Lernsubjekts, dessen Existenzialität darin besteht, optimierbar zu sein. Davon zeugt der von transnationalen Akteurinnen und Akteuren forcierte Diskurs zu $21^{\text {st }}$-Century-Skills, der Aspekte wie Kreativität, Kommunikation und kritische Problemlösung zu den entscheidenden Kompetenzen

5 In diesem Sinne diskutiert etwa Martin Karcher in seinem Beitrag anhand der Prüfung die durch Datafizierung und Digitalisierung evozierten Verschiebungen des epistemischen Zugriffs auf die Erzeugung eines pädagogisch relevanten spezifischen Wissens und auf das pädagogische Feld im Allgemeinen. 
erklärt, die in Schule und Hochschule entwickelt werden sollten. ${ }^{6}$ Ganz ähnlich ist das sogenannte Manifest zu «New Learning» gelagert, das im vergangenen Jahr von der Fernuniversität Hagen initiiert wurde (HM 2020). Es handelt sich um Diskurse, in denen über die Erstellung von und mittels Daten gelernt wird; zugleich treten fachwissenschaftliche bzw. fachbezogene Bezüge in den Hintergrund.

Mit dem Einsatz datengetriebener Instrumente, die das Lernen überwachen, werden die beteiligten Akteurinnen und Akteure - Lehrende wie Lernende - im Hinblick auf ihre Optimierbarkeit adressiert, wobei für die Logik der Optimierbarkeitsadressierung gilt, dass die Möglichkeit zur Optimierung einer Aufforderung gleichkommt, dieses Potenzial auch zu verwirklichen. Die Optimierbarkeit, welche die datengetriebenen Instrumente in Aussicht stellen, ist dabei sehr unterschiedlich gelagert.

Zum Ersten richtet sich das Versprechen einer datengestützten Optimierung wie oben bereits angedeutet - auf einen höheren Leistungsoutput der Lernenden, Lehrenden und Bildungsinstitutionen, der durch eine Individualisierung des Lernens ermöglicht werden soll. Diese stärkere Individualisierung des Lernens durch die Möglichkeiten von Digitalisierung wird einerseits über die Flexibilisierung von Lehrangeboten realisiert, die in einen engen Zusammenhang mit heterogenitätssensibler und barrierefreier Lehre gestellt wird. Andererseits ist die Vorstellung einer zunehmend individualisierten Lehr-Lern-Situation an die Möglichkeiten geknüpft, individuelle Potenziale qua Berechnung vorherzusagen.

Aufgrund von Daten oder algorithmischen Berechnungen lassen sich individuelle Vorhersagen zu Lernerfolgen oder möglichen Lernschwierigkeiten treffen. Dienlich sind solcherlei Ergebnisse der predictive analytics sowohl für die Lernenden und Lehrenden als auch für das effektive Wirtschaften der Bildungsinstitutionen selbst: Angesichts der bestehenden Finanzierungsprobleme haben bspw. Hochschulen ein grosses Interesse an datengestützten Instrumenten, mit denen es gelingen könnte, Abbruchquoten zu senken (Antunes 2010) oder Studieninteressierte im Hinblick auf ihren prospektiven Studienerfolg zu beraten (Thompson, Schröder, und Wrana 2021). Mithilfe der Daten, die Studierende beim Ausfüllen eines Online-Studienwahl-Tests erzeugen, lassen sich bspw. Wahrscheinlichkeiten künftiger Erfolge oder Misserfolge im gewählten Studienbereich berechnen. Die datengestützte Optimierung lässt sich hier also im Rahmen einer «production of prediction» (Mackenzie 2015) lesen, die vornehmlich die Realisierung oder Abwendung der berechneten Vorhersage anvisiert. Die prospektiven Studierenden sollen sich bspw. im Hinblick auf die Anforderungen der Universität entweder als (passend) verstehen - oder an sich arbeiten, um die bestmögliche Compliance mit der Universität herzustellen (Thompson, Schröder, und Wrana 2021).

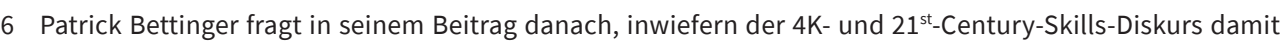
neue normative Ordnungen in das pädagogische Handeln einführt, die das Lernen vornehmlich in Richtung ökonomischer Kriterien wie wirtschaftlicher Verwertbarkeit ausrichten. 
In diesen Optimierungsmodus fällt auch das von Bröckling (vgl. 2012, 2020) beschriebene präventive Handeln. Durch die datengestützte oder algorithmische Vorhersage der wahrscheinlichen Zukunft wird es möglich, Probleme vorherzusagen, bevor sie überhaupt entstehen; eine datengestützte Selbstoptimierung wird sich darauf konzentrieren müssen, diese Vorhersage durch ein präventives Handeln abzuwenden und damit «die negativen Zukunftserwartungen zu widerlegen» (Bröckling 2012, 94). Wie oben bereits ausgeführt, fehlt in der Optimierung die Positivierung des Ziels - das (Optimum〉 verflüssigt sich. Aus diesem Grund kann die Vermeidung des Drohszenenarios prinzipiell grenzenlos werden. Konkret am oben genannten Beispiel der Beratung von Studieninteressierten: Die Passung zu einer Universität oder einem Studiengang führt nie zu einer Kongruenz, sodass sich die Passung nie erfüllt. ${ }^{7}$

Zum Zweiten lässt sich zeigen, dass die datengestützten Lern- und Prüfungsarchitekturen mitwirken, den Alltag von Lehrenden und Lernenden neu zu organisieren und Orientierungsfunktionen, Effektivierung und Vereinfachung, Routinisierung bieten können. Das schulische Arbeiten in Cloud-Systemen bspw. ermöglicht den Lehrenden wie den Lernenden nicht nur ein synchrones und gemeinsames Tätigsein, es schafft auch neue Formen der Systematisierung von und des Zugangs zu Informationen. Durch Digitalisierungsprozesse lassen sich Arbeitsabläufe optimieren, die v. a. bei den Lehrenden zu beobachten sind und die berufliche Entlastungen schaffen. ${ }^{8}$

Zugleich wird deutlich, dass mit einer zunehmenden Digitalisierung schulischer Praktiken auch vormalige Desiderata aufgegriffen werden, darunter die Hoffnung, Leistungen objektiv, reliabel und valide zu identifizieren. Lehrkräften und Dozierenden wird in Aussicht gestellt, durch datengestützte Unterrichtspraktiken Fehlerquellen zu entkommen, die in der 'sozialen Praxis) der Beurteilung von Prüfungen nicht vermeidbar sind. ${ }^{9}$ Wie sich dadurch ihr pädagogisches Selbstverständnis wandelt und welche Effekte dies für die ‘Sozialität des Lernens und Bewertens` hat, ist indes eine ganz eigene Frage.

7 Trotzdem muss wohl unterschieden werden zwischen einerseits solchen Optimierungsimperativen, die auf die (Freiwilligkeit) und Bindung der Akteurinnen und Akteure angewiesen sind und mit denen sich ein gewisses Mass an Glück versprochen wird (Foucault), und der Optimierung als Vorsorge andererseits: «Dispositive der Angst und Sorge operieren anders als jene, die den Ehrgeiz kitzeln oder mit Versprechen von Erfolg, Wohlstand und Gesundheit locken» (Bröckling 2020, o. S.).

8 Diesen Aspekt aufnehmend zeigen Sieglinde Jornitz und Felicitas Macgilchrist in ihrem Beitrag die Ambivalenzen auf, die Digitalisierungsprozesse in der Schule für das Handeln von Lehrpersonen und Lernenden aufweisen. Durch die im Unterricht implementierten digitalen Tools werden neue Formen der Sichtbarkeit etabliert, die zugleich motivieren und kontrollieren.

9 Sabrina Schröder zeigt in ihrem Beitrag, wie mit Bezug auf Datafizierungsprozesse das Versprechen verbunden wird, die seit Jahren diskutierten Probleme der Leistungsbewertung bzw. deren Fragwürdigkeit zu bearbeiten oder gar zu überwinden. Diesbezügliche Hoffnungen ergeben sich v. a. auf der Ebene der Prognose von Schulleistung, der Vermeidung subjektiver Fehlerquellen beim Beurteilen und einer Versöhnung des Streits um die Frage nach der standardisierten oder individualisierten Leistungsmessung. 
Aus einer bildungstheoretischen Sicht ist zum Dritten daran zu erinnern, wie eine datengestützte Optimierung das moderne ${ }^{10}$ Versprechen von Fortschritt und Verbesserung neuformatiert, indem über die Verknüpfung des Menschen mit seinen (vielen) Daten eine postmediale Konstellation entsteht, welche weitreichende Veränderungen des Menschen als Lebewesen impliziert: politisch, gesellschaftlich, sozial und in Bezug auf seine (Natur`. Unter der Einsicht, dass (Daten` zur neuen Währung im informationellen Kapitalismus (Castells) bzw. im knowledge-intensive capitalism werden, ist von einer zunehmenden ökonomischen Durchdringung von Bildungs-, Lern- und Erziehungsprozessen auszugehen (Amos 2019). Zugleich wirft dieser ¿Umbau des Menschen vermittelt über Daten` aus einer kritisch posthumanistischen ${ }^{11}$ Sicht zahlreiche Fragen auf.

Dass Daten zu einer vermittelnden Grundlage für die Veränderung von Personen wie für die Weiterentwicklung von pädagogischen Institutionen und von Wissenschaftszweigen werden, dass sie zugleich zum basalen Tauschmittel avancieren (und damit immer auch ausbeutbar sind), verdeutlicht, wie grundsätzlich die Produktion pädagogischen Wissens von den skizzierten Entwicklungen betroffen ist. In den vergangenen Jahren haben die Critical Data Studies die Konsequenzen dieser Entwicklungen für die Regierung der Subjekte herausgestellt (vgl. hierfür bspw. Williamson 2017; Kitchin 2014; Kitchin und Lauriault 2014) und dabei auf den Einfluss privatwirtschaftlicher Akteurinnen und Akteure hingewiesen, die wiederum auf eine Optimierung von Bildungstechnologien und Dateninfrastrukturen drängen. Letztere bleiben den Nutzerinnen und Nutzern zunehmend verborgen, sodass die Grenzen und Wege von Datenströmen kaum mehr überschaut werden können.

Angesichts dieser Diffusion wird es zukünftig wichtig sein, erziehungswissenschaftliche Analysen und Critical Code Studies zu verbinden. Es muss danach gefragt werden, wie die digitalen Transformationen im Erziehungs- und Bildungswesen auf die Höhe einer reflexiven Erziehungswissenschaft gebracht werden können. Darunter sind theoretisch informierte Auseinandersetzungen ebenso wie disziplinpolitische Stellungnahmen zu verstehen. ${ }^{12}$ Die folgenden grundlegenden Fragen, welche

10 Christiane Thompson und Estella Ferraro verdeutlichen in ihrem Beitrag, wie gerade das moderne Selbstverhältnis von dieser Neuformatierung betroffen ist: Die Suche nach dem Selbst spannt sich auf zwischen der Möglichkeit einer datenvermittelten Selbstbestimmung bzw. -optimierung und dem kontinuierlichen Scheitern dieses Versuchs. In dieser Bewegung zwischen Identifikation und Versagung wird die Frage nach dem Selbst zum Motor der datengestützten Selbstoptimierung.

11 Darunter fällt die Frage nach der «Stossrichtung` oder inhaltlichen Ausgestaltung dessen, was als «Verbesserung des Menschen) gelten soll oder welche gegenwärtigen und zukünftigen Visionen des Menschen mittels der Rhetorik einer prinzipiellen Mess- und Optimierbarkeit im Zusammenhang mit einem unaufhörlichen Technologiefortschritt auf diese Weise transportiert werden. Dies ist Thema des Beitrags von Dan Verständig.

12 Fenwick und Edwards (2016) diskutieren unterschiedliche Weisen einer kritischen Stellungnahme in Bezug auf die Ausbildung und Professionalisierung angehender Pädagoginnen und Pädagogen. Auch dieser Aspekt ist u. E. Teil der anstehenden systematisch erziehungswissenschaftlichen Auseinandersetzung. 
auf das Verhältnis von (Selbst-)Optimierung und Digitalisierung von Erziehung und Bildung zielen und die Leitfragen des vorliegenden Themenheftes bilden, werden dabei zu berücksichtigen sein:

- Wie verändern sich Selbst- und Weltverhältnisse angesichts der unterschiedlichen Formen und Praxen datengestützter Optimierung? Wie sind diese Veränderungen im Verhältnis zur Konstitution pädagogischen Wissens einzuordnen?

- Wie lassen sich datengestützte Optimierungsprozesse praktiken- und subjektivierungstheoretisch erschliessen und wie sind diese im Hinblick auf den öffentlichen Bildungsauftrag oder auf die Aufgabe der Leistungsüberprüfung zu reflektieren?

- Wie verändern sich Regierungsweisen, Kontrollformen und Sichtbarkeitsregime in pädagogischen Praktiken durch Digitalisierungs- und Datafizierungsprozesse? Was sind machttheoretische Effekte dieser Transformationen und wie wirken diese konkret in den pädagogischen Handlungsfeldern?

- Wie lassen sich datengestützte Optimierungsprozesse oder eine potenzielle <Regierung des Pädagogischen unter digitalisierten Vorzeichen> einer kritischen Analyse unterziehen?

\section{Zu den Beiträgen des Themenheftes}

Dan Verständig untersucht Prozesse der Selbstoptimierung aus einer post- bzw. transhumanistischen Perspektive, genauer: im Hinblick auf die Nutzung digitaler Technologien zur Berechnung und Vermessung des Menschen im Zusammenhang der Überwindung seiner Mangelhaftigkeit. Der Autor macht deutlich, wie das Denken über digitale Technologien in die Handlungsvollzüge der Menschen eingeschrieben wird. Angesichts der Einsicht, dass der Markt der Selbstoptimierung von der Aggregation grosser Datenmengen und deren Rekontextualisierung abhängig ist, erscheint die Optimierung des Menschen durch technologischen Fortschritt zunehmend alternativlos.

In ihrem Beitrag diskutieren Christiane Thompson und Estella Ferraro, inwiefern Praktiken der datengestützten Selbstoptimierung unter modernisierungstheoretischen Vorzeichen zu den Konstitutionsbedingungen des Selbst gehören. Die Datafizierung tritt zur Fragmentierung und Verflüssigung des modernen Lebens in ein doppeltes Verhältnis: Erstere nährt die Hoffnung, dass das Selbst doch noch zu einer finalen Bestimmung gelangen könnte. Zugleich erneuert sich im Medium der Daten der symbolische Riss mit der Folge der Entzogenheit des Selbst. Der Beitrag reflektiert die bildungstheoretischen Konsequenzen dieser Dialektik von Bestimmung und Unbestimmbarkeit. 
Am Forschungsgegenstand der im angloamerikanischen Raum etablierten $21^{\text {st }}$ Century- bzw. 4K-Skills analysiert Patrick Bettinger unter gouvernementalitätstheoretischer Perspektive eine neue Spielart pädagogischer Führungsverhältnisse im Bildungsbereich. Er zeigt, wie sich die digitale Reformierung des Bildungssystems mit der Produktion neuer pädagogisch-politischer Steuerungstechnologien und v. a. verwertungslogisch ausgerichteter Subjektivierungs- und Normalisierungsanforderungen verschränkt. Darüber hinaus wird deutlich, dass die hegemoniale Definitionsmacht bezüglich der inhaltlichen Ausgestaltung von Bildungszielen vermehrt global agierenden Netzwerken aus bildungspolitischen Akteurinnen und Akteuren und einer Vielzahl an Unternehmen und privatwirtschaftlichen, nichtstaatlichen Organisationen zugesprochen wird, die «das Pädagogische` wirkmächtig regieren.

Sabrina Schröder diskutiert anhand des neuen Forschungsfeldes der Learning Analytics, wie mit dem Einsatz digitaler Lerntools das Versprechen verbunden werden kann, Lernprozesse und insbesondere Lernoutputs zu optimieren. Mit den Tools werden auf diese Weise Probleme bearbeitbar, die v. a. im Bereich der Schulpädagogik schon seit Langem prozessiert werden, wie die Frage nach der Objektivität von Leistungsmessungen oder die Möglichkeit von Lernprognosen. Der Beitrag zeigt, dass mit diesem Versprechen Komplexitätsreduktionen in Bezug auf das Lernsubjekt und -objekt einhergehen, die wiederum einen Verantwortungsverlust der pädagogisch Handelnden mit sich bringen.

Martin Karcher legt den Wandel pädagogischer Formen und Wirkungen von Prüfungen vor dem Hintergrund der Datafizierung des Pädagogischen dar. In zunehmendem Masse würden sich Praktiken des Prüfens dahingehend entwickeln, dass weniger die Erhebung als vielmehr die Generierung von Wissen in deren Mittelpunkt stehe. Auf diese Weise erfolge über die Prüfung eine digital transformierte Kartografie des Pädagogischen selbst. These des Beitrags ist, dass damit ein neues digitales pädagogisches Wirklichkeitsmodell entstehe - der Autor spricht von einem «data realism» -, das zur Grundlage pädagogischen Denkens und Handelns wird und fundamentale Verschiebungen dahingehend anzeigt, welches Wissen von der Pädagogik künftig erwartet werden darf.

Sieglinde Jornitz und Felicitas Macgilchrist analysieren, wie digitale Tools zu neuen Formen der Sichtbarkeit innerhalb und ausserhalb des Unterrichts und zu Transformationen schulischer Praktiken führen. Über die Analyse eines Interviews mit einer Lehrerin, die digitale Tools nutzt, rekonstruieren sie drei unterschiedliche Sichtbarkeitsverschiebungen: im Hinblick auf pädagogische Praktiken, auf die 
Verhältnisse der Schülerinnen und Schüler untereinander und auf die ökonomisch nutzbare Abschöpfung von Daten insgesamt - mit weitreichenden Folgen für Demokratie, Freiheit und Wissen im Rahmen individueller und kollektiver Entscheidungsfindung.

\section{Literatur}

Amos, Karin. 2019. "Digitization, Disruption, and the (Society of Singularities): The Transformative Power of the Global Education Industry». In Researching the Global Education Industry, herausgegeben von Marcelo Parreira do Amaral, Gita Steiner-Khamsi, und Christiane Thompson, 225-249. Basingstoke: Palgrave Macmillan.

Antunes, Cláudia. 2010. «Anticipating Students' Failure As Soon As Possible». In Handbook of Educational Data Mining, herausgegeben von Cristóbal Romero, Sebastian Ventura, Sebastian, Mykola Pechenizkiy, Ryan Baker, 353-363. Boca Raton, FL: CRC Press.

Baumert, Jürgen, und Klaus-Jürgen Tillmann, Hrsg. 2017. «Empirische Bildungsforschung. Der kritische Blick und die Antwort auf die Kritiker». Zeitschrift für Erziehungswissenschaft Sonderheft 31. https://doi.org/10.1007/978-3-658-13785-4.

Bellmann, Johannes. 2006. «Bildungsforschung und Bildungspolitik im Zeitalter «Neuer Steuerung»». Zeitschrift für Pädagogik 52 (4): 487-504. https://doi.org/10.25656/01:4468.

Bellmann, Johannes. 2016. "Datengetrieben und/oder evidenzbasiert?». Zeitschrift für Erziehungswissenschaft (Sonderheft) 31 (4): 147-161. https://doi.org/10.1007/978-3-658-137854_9.

Bellmann, Johannes, Dirk Braun, Martina Diedrich, Katharina Maag Merki, Marcelo Parreira do Amaral, und Kate Maleike. 2020. «Wer steuert die Bildung - Wer steuert die Schule? Ein öffentliches Podiumsgespräch zur Eröffnung des 26. Kongresses der Deutschen Gesellschaft für Erziehungswissenschaft (DGfE) am 18. März 2018 in Essen». In Bewegungen. Beiträge zum 26. Kongress der Deutschen Gesellschaft für Erziehungswissenschaft, herausgegeben von Isabell van Ackeren, Helmut Bremer, Fabian Kessl, Hans-Christoph Koller, Nicolle Pfaff, Caroline Rotter, Dominique Klein, und Ulrich Salaschek, 175-194. Opladen/Berlin/Toronto: Barbara Budrich. https://doi.org/10.3224/84742385.

Bellmann, Johannes, und Thomas Müller, Hrsg. 2011. Wissen, was wirkt. Kritik evidenzbasierter Pädagogik. Wiesbaden: VS. https://doi.org/10.1007/978-3-531-93296-5.

BMBF. 2016. Bildungsoffensive für die digitale Wissensgesellschaft. Strategie des Bundesministeriums für Bildung und Forschung. Berlin. https://www.kmk.org/fileadmin/pdf/Themen/ Digitale-Welt/Bildungsoffensive_fuer_die_digitale_Wissensgesellschaft.pdf.

BMBF. 2021. «Bekanntmachung des BMBF 〈KI in der Hochschulbildung»». https://www.bmbf. de/bmbf/shareddocs/bekanntmachungen/de/2021/02/3409_bekanntmachung.html.

Boltanski, Luc, und Ève Chiapello. 2003. Der neue Geist des Kapitalismus. Konstanz: Herbert von Halem Verlag, UVK. 
Bröckling, Ulrich. 2005. «Projektwelten. Anatomie einer Vergesellschaftungsform». Leviathan 33 (3): 364-383. https://www.jstor.org/stable/23987399.

Bröckling, Ulrich. 2007. Das unternehmerische Selbst. Soziologie einer Subjektivierungsform. Frankfurt a. M.: Suhrkamp.

Bröckling, Ulrich. 2012. «Dispositive der Vorbeugung: Gefahrenabwehr, Resilienz, Precaution». In Sicherheitskultur. Soziale und politische Praktiken der Gefahrenabwehr, herausgegeben von Christopher Daase, Philipp Offermann, und Valentin Rauer, 39-108. Frankfurt/New York: Campus.

Bröckling, Ulrich. 2013. «In der Optimierungsfalle. Zur Soziologie der Wettbewerbsgesellschaft». Supervision 31 (4): 4-11.

Bröckling, Ulrich. 2020. "Optimierung, Preparedness, Priorisierung. Soziologische Bemerkungen zu drei Schlüsselbegriffen der Gegenwart». Soziopolis - Gesellschaft beobachten. online unter: https://www.soziopolis.de/beobachten/gesellschaft/artikel/optimierungpreparedness-priorisierung/.

Casale, Rita, Charlotte Röhner, Andreas Schaarschuch, und Heinz Sünker. 2010. «Entkopplung von Lehrerbildung und Erziehungswissenschaft: Von der Erziehungswissenschaft zur Bildungswissenschaft». Erziehungswissenschaft 21 (41): 43-66. https://doi. org/10.25656/01_4028.

DGfE-Kongress (DGfE 2020). https://dgfe2020.uni-koeln.de.

Fenwick, Tara, und Richard Edwards. 2016. «Die Auswirkungen digitaler Technologien auf professionelle Verantwortung und Ausbildung». Berliner Debatte Initial 27 (1): 6-21.

FernUniversität Hagen (HM 2020). 2020. Lernen neu denken. Das Hagener Manifest zu New Learning. https://www.fernuni-hagen.de/imperia/md/content/universitaet/hagenermanifest/ hagener-manifest.pdf.

Foucault, Michel. 1993. «Technologien des Selbst». In Technologien des Selbst, herausgeben von Luther H. Martin, Huck Gutman, und Patrick H. Hutton, 24-62. Frankfurt a. M.: S. Fischer.

Foucault, Michel. 2004. Geschichte der Gouvernementalität I. Sicherheit - Territorium - Bevölkerung. Vorlesungen am Collège de France 1977-1978. Frankfurt a. M.: Suhrkamp.

Kitchin, Rob. 2014. «Big Data, New Epistemologies and Paradigm Shifts». Big Data and Society 1 (1): 1-12. https://doi.org/10.1177/2053951714528481.

Kitchin, Rob, und Tracey P. Lauriault. 2014. Towards critical data studies: Charting and unpacking data assemblages and their work. The Programmable City Working Paper 2. http:// mural.maynoothuniversity.ie/5683/1/KitchinLauriault_CriticalDataStudies_ProgrammableCity_WorkingPaper2_SSRN-id2474112.pdf.

KMK. 2016. Bildung in der digitalen Welt. Strategie der Kultusministerkonferenz. Berlin. https:// www.kmk.org/fileadmin/Dateien/pdf/PresseUndAktuelles/2017/Strategie_neu_2017_datum_1.pdf.

Mackenzie, Adrian. 2015. "The production of prediction: What does machine learning want?» European Journal of Cultural Studies 18 (4-5): 429-445. https://doi. org/10.1177/1367549415577384. 
Mayer, Ralf, und Christiane Thompson. 2013. «Inszenierung und Optimierung des Selbst. Eine Einleitung». In Inszenierung und Optimierung des Selbst. Zur Analyse gegenwärtiger Selbsttechnologien, herausgegeben von Ralf Mayer, Christiane Thompson, und Michael Wimmer, 7-28. Wiesbaden: VS. https://doi.org/10.1007/978-3-658-00465-1.

OECD. 2002. Understanding the Brain: The Birth of a Learning Science. https://www.oecd-ilibrary. org/education/understanding-the-brain-the-birth-of-a-learning-science_9789264029132en.

Reckwitz, Andreas. 2006. Das hybride Subjekt. Eine Theorie der Subjektkulturen von der bürgerlichen Moderne zur Postmoderne. Weilerswist: Velbrück.

Ricken, Norbert. 2006. Die Ordnung der Bildung. Beiträge zu einer Genealogie der Bildung. Wiesbaden: VS Verlag für Sozialwissenschaften. https://10.1007/978-3-531-90474-0.

Ricken, Norbert, Rita Casale, und Christiane Thompson, Hrsg. 2019. Subjektivierung. Erziehungswissenschaftliche Theorieperspektiven. Weinheim/Basel: Beltz Juventa. https://doi. org/10.30965/25890581-0901054.

Röcke, Anja. 2021. Soziologie der Selbstoptimierung. Berlin: Suhrkamp.

Romero, Cristobal, Sebastian Ventura, Mykola Pechenizkiy, und Ryan Baker, Hrsg. 2010. Handbook of Educational Data Mining. Boca Raton: CRC Press.

Thompson, Christiane. 2004. «Diesseits von Authentizität und Emanzipation. Verschiebungen kritischer Erziehungswissenschaft zu einer «kritischen Ontologie der Gegenwart»». In Michel Foucault: Pädagogische Lektüren, herausgegeben von Nobert Ricken, und Markus Rieger-Ladich, 39-56, Wiesbaden: VS Verlag für Sozialwissenschaften. https://10.1007/978-3-322-85159-8_3.

Thompson, Christiane. 2019. «Bildung in Zeiten globaler Expertise». In Subjektivierung. Erziehungswissenschaftliche Theorieperspektiven, herausgegeben von Norbert Ricken, Rita Casale, und Christiane Thompson, 281-299. Weinheim: Beltz Juventa. https://doi. org/10.30965/25890581-0901054.

Thompson, Christiane. 2021: «Praktiken und Formen der Theorie. Konturen der Wissensarbeitsforschung». In Praktiken und Formen der Theorie. Perspektiven der Bildungsphilosophie, herausgegeben von Christiane Thompson, Malte Brinkmann, und Markus Rieger-Ladich, 11-31. Weinheim: Beltz Juventa.

Thompson, Christiane, Sabrina Schröder, und Daniel Wrana. 2021. «Fostering the «Promising Student> at the Outset. The Digitization and Management of Student Success in the Competitive University». In Geopolitical Transformations in Higher Education. Imagining, Fabricating and Contesting Innovation, herausgegeben von Marcelo Parreira do Amaral, und Christiane Thompson. Farmington Hills: Springer (i. D.).

Williamson, Ben. 2017. Big Data in Education. The digital future of learning, policy and practice. Los Angeles u. a.: Sage. https://doi.org/10.1080/09620214.2019.1690546. 


\section{Beiträge}

Bettinger, Patrick. 2021. «Etablierung normativer Ordnungen als Spielarten optimierter Selbstführung? Die Regierung des Pädagogischen am Beispiel des 4K- und 21st-Century-SkillsDiskurses». Herausgegeben von Estella Ferraro, Sabrina Schröder, und Christiane Thompson. MedienPädagogik: Zeitschrift für Theorie und Praxis der Medienbildung 45 (Pädagogisches Wissen): 34-58. https://doi.org/10.21240/mpaed/45/2021.12.17.X.

Jornitz, Sieglinde, und Felicitas Macgilchrist. 2021. «Datafizierte Sichtbarkeiten: Vom Panopticon zum Panspectron in der schulischen Praxis». Herausgegeben von Estella Ferraro, Sabrina Schröder, und Christiane Thompson. MedienPädagogik: Zeitschrift für Theorie und Praxis der Medienbildung 45 (Pädagogisches Wissen): 98-122. https://doi.org/10.21240/ mpaed/45/2021.12.21.X.

Karcher, Martin. 2021. «Das Ende der Prüfung als Anfang des «Data Realism〉? Erkundungen zur Digitalisierung pädagogischen Wissens». Herausgegeben von Estella Ferraro, Sabrina Schröder, und Christiane Thompson. MedienPädagogik: Zeitschrift für Theorie und Praxis der Medienbildung 45 (Pädagogisches Wissen): 80-97. https://doi.org/10.21240/ mpaed/45/2021.12.19.X.

Schröder, Sabrina. 2021. «Vom pädagogischen Misstrauen gegen die Prüfung und dem Versprechen einer digitalen Problemlösung». Herausgegeben von Estella Ferraro, Sabrina Schröder, und Christiane Thompson. MedienPädagogik: Zeitschrift für Theorie und Praxis der Medienbildung 45 (Pädagogisches Wissen): 59-79. https://doi.org/10.21240/ mpaed/45/2021.12.18.X.

Thompson, Christiane, und Estella Ferraro. 2021. «Zur datengestützten Selbstoptimierung aus bildungstheoretischer Sicht». Herausgegeben von Estella Ferraro, Sabrina Schröder, und Christiane Thompson. MedienPädagogik: Zeitschrift für Theorie und Praxis der Medienbildung 45 (Pädagogisches Wissen): 19-33. https://doi.org/10.21240/mpaed/45/2021.12.16.X.

Verständig, Dan. 2021. «Die besten Menschen, die es jemals gab: Die Rede von der Verbesserung des Menschen durch digitale Technologien und ihre Auswirkungen auf das Soziale». Herausgegeben von Estella Ferraro, Sabrina Schröder, und Christiane Thompson. MedienPädagogik: Zeitschrift für Theorie und Praxis der Medienbildung 45 (Pädagogisches Wissen): 1-18. https://doi.org/10.21240/mpaed/45/2021.12.15.X. 